\title{
Fundamental parameters and evolutionary state of the Herbig Ae star candidate HD 35929
}

\author{
A. S. Miroshnichenko ${ }^{1,2,3}$, R. O. Gray ${ }^{4}$, V. G. Klochkova ${ }^{5,6}$, K. S. Bjorkman ${ }^{2}$, and K. S. Kuratov ${ }^{7}$ \\ 1 Max-Planck-Institut für Radioastronomie, Auf dem Hügel 69, 53121 Bonn, Germany \\ e-mail: anatoly@physics.utoledo.edu \\ 2 Ritter Observatory, Dept. of Physics \& Astronomy, The University of Toledo, Toledo, OH 43606-3390, USA \\ 3 Pulkovo Observatory, Saint-Petersburg, 196140, Russia \\ ${ }^{4}$ Dept. of Physics and Astronomy, Appalachian State University, Boone, NC 28608, USA \\ 5 Special Astrophysical Observatory of the Russian Academy of Sciences, Karachai-Cirkassian Republic, \\ Nizhnij Arkhyz, 369167, Russia \\ ${ }^{6}$ Isaac Newton Institute of Chile, SAO Branch, Russia \\ ${ }^{7}$ Fesenkov Astrophysical Institute, Almaty 480068, Kazakhstan
}

Received 8 July 2004 / Accepted 27 July 2004

\begin{abstract}
HD 35929 is a pre-main-sequence Herbig Ae star candidate that exhibits emission in the $\mathrm{H} \alpha$ line and a weak IR excess. Although its luminosity type was estimated as IV from low-resolution spectroscopy, the insignificant HIPPARCOS parallax $(0.88 \pm 0.93$ mas) contradicted the low luminosity. To resolve this discrepancy, we took 3 high-resolution optical spectra of the star (region 5200-6915 $\AA, R \sim 60000$ ) and used an archival one (4000-6800,$R \sim 48000$ ). Our analysis of the spectral and photometric information shows that HD 35929 is an F2 III star with $T_{\text {eff }}=6880 \pm 100 \mathrm{~K}, \log g=3.3 \pm 0.1$, $\log L / L_{\odot}=1.7 \pm 0.2, v \sin i=70 \pm 5 \mathrm{~km} \mathrm{~s}^{-1}, M=2.3 \pm 0.2 M_{\odot}$, a mild metal deficit $[\mathrm{Fe} / \mathrm{H}]=-0.2 \pm 0.1$, and a weak interstellar reddening $\left(A_{\mathrm{V}} \sim 0.1 \mathrm{mag}\right)$ at a distance $D=345 \pm 60 \mathrm{pc}$. These results confirm an earlier suggestion by Marconi et al. (2000) that HD 35929 is located within the instability strip. At the same time, we argue that it is not a young object, but rather a post-main-sequence giant. The fundamental parameters and IR excess of HD 35929 are similar to those of HD 19993, an emission-line late A-type giant recently discovered by us (Miroshnichenko et al. 2003). These objects might represent a group of evolved intermediate-mass stars with an enhanced mass loss.
\end{abstract}

Key words. techniques: spectroscopic - techniques: photometric - stars: circumstellar matter - stars: individual: HD 35929

\section{Introduction}

Balmer line emission and large infrared (IR) excess are inevitable features of pre-main-sequence stars. They are often used to select candidates for this type of object. At the same time, evolved stars may display such features as well. Therefore, only careful analysis of high quality multiwavelength and multitechnique data will permit conclusive classification.

Since 1999 we have been conducting high-resolution optical spectroscopic observations of early-type stars with IR excesses detected by the IRAS satellite. These data are supported by low-resolution optical and IR spectroscopy and multicolor photometry in most cases. The program's main goal is to determine accurate fundamental parameters of the underlying stars and search for new criteria to separate young and evolved stars with circumstellar (CS) envelopes.

So far two results of this program have been published. Studying the pre-main-sequence candidate star IP Per, we found that it is indeed a young late A-type metal-deficient star (Miroshnichenko et al. 2001). The second result concerned 2 newly found emission-line objects, HD 19993 and HD 29035. We showed that HD 29035 is a pre-main-sequence B9-type candidate, while HD 19993 is a metal-deficient A7type giant (Miroshnichenko et al. 2003). In this paper we report the results of our spectroscopic and photometric study of another pre-main-sequence candidate star, HD 35929.

The IR excess in HD 35929 was detected by IRAS and first reported by Oudmaijer et al. (1992), who listed it as a Herbig Ae/Be star candidate. Later it appeared in the list of Herbig Ae/Be stars and candidate members by Thé et al. (1994, their Table 1) and was investigated as a member of the group in a number of follow-up studies. Grady et al. (1996) reported emission in the UV Mg II, C IV, and O I lines, which is also observed in the spectra of other Herbig Ae stars. Based on the broad wings of the Mg II line, these authors estimated the projected rotational velocity of HD 35929 to be between 150 and $200 \mathrm{~km} \mathrm{~s}^{-1}$. They also reported a single-peaked emission $\mathrm{H} \alpha$ line and higher Balmer series members in absorption. The IUE data were found to be consistent with a spectral type 
Table 1. Log of spectroscopic observations.

\begin{tabular}{crrlcclc}
\hline \hline Date & MJD & Obs. & $\begin{array}{l}\text { Telescope/ } \\
\text { Spectrometer }\end{array}$ & $\begin{array}{c}\text { Sp. Range } \\
\AA\end{array}$ & $R$ & $\begin{array}{l}\text { Exp. } \\
\text { time }\end{array}$ & $\begin{array}{c}V_{\mathrm{r}} \\
\mathrm{km} \mathrm{s}^{-1}\end{array}$ \\
\hline $1998 / 01 / 27$ & 840.81 & OHP & 1.93-m/ELODIE & $4000-6815$ & 48000 & $1 \mathrm{~h}$ & $19.6 \pm 4.6$ \\
$2001 / 10 / 10$ & 2192.94 & McD & 2.1-m/Sandiford & $5540-6910$ & 60000 & $10 \mathrm{~min}$. & $22.4 \pm 3.8$ \\
$2002 / 09 / 28$ & 2545.99 & McD & 2.1-m/Sandiford & $5430-6830$ & 60000 & $10 \mathrm{~min}$. & $21.5 \pm 3.6$ \\
$2003 / 02 / 23$ & 2694.20 & SAO & 6-m/NES & $5100-6580$ & 60000 & $2 \mathrm{~h}$ & $19.6 \pm 4.4$ \\
\hline
\end{tabular}

The modified Julian dates (MJD = JD-2 450000) are shown in Col. 2; the observatories in Col. 3 are Observatoire de Haute-Provence (OHP, France), McDonald Observatory (McD, USA), and Special Astrophysical Observatory of the Russian Academy of Sciences (SAO, Russia); the spectral resolving power $R=\Delta \lambda / \lambda$ in Col. 6; and the average radial velocity determined using unblended spectral lines in Col. 9.

The ELODIE spectrograph is described in Corporon \& Lagrange (1999), Sandiford in McCarthy et al. (1993), and NES in Panchuk et al. (2002).

of A8-F0 with a total reddening of $E(B-V)=0.10-0.12 \mathrm{mag}$, of which $0.06 \mathrm{mag}$ is interstellar.

Another pre-HIPPARCOS study by Miroshnichenko et al. (1997a) suggested that HD 35929 is a young A8 ve star with $E(B-V)=0.15 \mathrm{mag}$ located at a distance of $\sim 100 \mathrm{pc}$ from the Sun. The distance estimate was solely based on the weak IR excess, which is characteristic of young objects close to the main-sequence. If this were the case, HIPPARCOS easily would have measured the object's parallax, which however turned out to be comparable with its uncertainty $(0.88 \pm$ 0.93 mas). From this result, van den Ancker et al. (1998) suggested that HD 35929 is a member of the Ori OB1 association at a distance $D=430 \mathrm{pc}$. They reported a spectral type F0 IIIe, an extinction $A_{\mathrm{V}}=0.40 \mathrm{mag}$, and a luminosity $\log L / L_{\odot} \geq 1.92$. These parameters put HD 35929 well above the main-sequence, close to the birthline for Herbig Ae/Be stars (Palla \& Stahler 1993).

Marconi \& Palla (1998) defined the instability strip for pre-main-sequence intermediate-mass stars and suggested HD 35929 as a candidate for pulsational brightness variations, which were found by Marconi et al. (2000). Thus, the object was classified a $\delta$ Scuti variable with a pulsational period of $0.196 \pm 0.005$ days. Considering this period to correspond to the pulsation in the first or second overtone, Marconi et al. (2000) calculated the star's mass to be 3.4 and $3.8 M_{\odot}$, respectively, for the pre-main-sequence evolutionary tracks and $2.7 M_{\odot}$ (second overtone only) for the post-main-sequence tracks. The corresponding values of the luminosity are 83 and $114 L_{\odot}$ for the pre-main-sequence and $83 L_{\odot}$ for the post-mainsequence solution. This result indirectly confirms a relatively high luminosity of the star.

Nevertheless, Gray \& Corbally (1998) classified the star as F2 IVe using low-dispersion spectroscopy in the region 3800-5600 A. This result emphasized the controversy between the apparently large distance (due to the small parallax), which implies a relatively young age (not far from the birthline), and the small IR excess. The latter is significantly lower than that of other Ae and Fe pre-main-sequence star candidates (e.g., Malfait et al. 1998).

Although HD 35929 is a bright star $(V=8.1 \mathrm{mag})$, no highresolution and high signal-to-noise spectroscopy has been presented in the literature. The only échelle optical spectra were mentioned by Grady et al. (1996), but these were not discussed in detail. In an attempt to resolve the problems discussed above, to determine the star's fundamental parameters and evolutionary state, we obtained optical spectra at high resolution and collected multiwavelength photometric data to aid the analysis.

In Sect. 2 we describe our observations, in Sect. 3 we present an analysis of the object's properties, and in Sect. 4 we discuss its nature and evolutionary state.

\section{Observations}

The four high-resolution spectra of HD 35929 we have were obtained at three different observatories with échelle spectrometers in 1998-2003. The observing log is presented in Table 1. The signal-to-noise ratio in the continuum exceeds 100 in all the spectra redward of $\sim 5000 \AA$. In the ELODIE spectrum, it is $\sim 30-40$ in the region $4000-4300 \AA$ and gradually increases towards longer wavelengths, reaching $\sim 100$ at $\sim 4800 \AA$.

The McDonald data were reduced in IRAF ${ }^{1}$. The SAO and OHP data were reduced in MIDAS.

\section{Results}

\subsection{Spectral line content}

All our spectra are virtually identical to each other. The only emission line in the observed wavelength region is a singlepeaked $\mathrm{H} \alpha$. Additionally, $\mathrm{H} \beta$ shows a slight filling in near the profile center (see Fig. 1). The spectrum contains numerous metallic lines and is very similar to that of normal early F-type stars (see Sect. 3.2 and Fig. 1). The radial velocities $\left(V_{\mathrm{r}}\right)$ were measured by fitting the line profiles to a Gaussian. The results presented in Table 1 reveal no $V_{\mathrm{r}}$ variations greater than the statistical uncertainty $\left(\sim 4 \mathrm{~km} \mathrm{~s}^{-1}\right)$ and give an average $V_{\mathrm{r}}=20.7 \pm 1.6 \mathrm{~km} \mathrm{~s}^{-1}$. No diffuse interstellar bands (DIB) were detected, suggesting that the interstellar reddening is small.

${ }^{1}$ IRAF is distributed by the National Optical Astronomy Observatories, which are operated by the Association of Universities for Research in Astronomy, Inc., under contract with the National Science Foundation. 

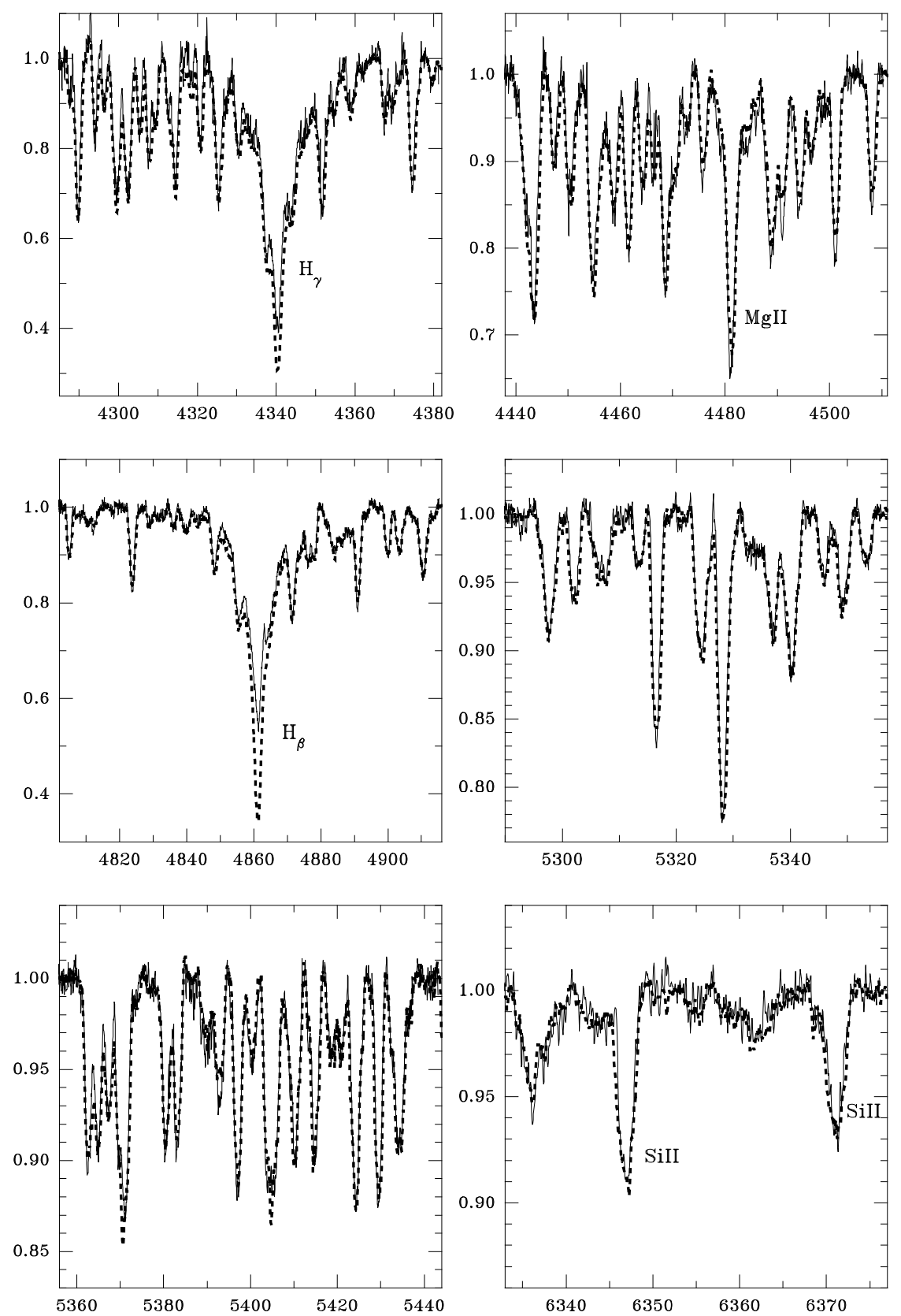

Fig. 1. Portions of the spectrum of HD 35929 (solid line). A comparison spectrum of $\beta$ Cas is shown by dotted lines. Most of the unmarked lines are those of Fe I and Fe II. The intensities are normalized to the underlying continuum, while the wavelengths are given in $\AA$.

\subsection{Fundamental parameters}

In the optical region, HD 35929 was observed in the following photometric systems: Johnson (Miroshnichenko et al. 1997a) and Johnson-Cousins UBVRI (de Winter et al. 2001), Strömgren $u v b y \beta$ (Olsen 1983; Perry 1991; Olsen 1994), Walraven (de Geus et al. 1990), and Vilnius (Eimontas \& Sudzius 1998). Although the star exhibits some brightness variations $\left(\Delta m_{\mathrm{pg}} \sim 0.5 \mathrm{mag}\right.$, Straizys $1963 ; \Delta V \sim 0.2 \mathrm{mag}$, Miroshnichenko et al. 1997a and de Winter et al. 2001), it primarily has been observed near the brightest state $(V=$ $8.12 \pm 0.02 \mathrm{mag}$ ) with the color indices shown in Table 2 . It is remarkable that the Walraven and Vilnius data obtained at very different epochs (1970-s and 1990-s, respectively) transformed into the Johnson system both give $B-V=$ $0.42 \mathrm{mag}$, which also coincides with the mean data by Miroshnichenko et al. (1997a) and de Winter et al. (2001).

The weak emission-line spectrum indicates that the circumstellar (CS) matter has a very small effect on the line strengths and color indices. Comparison with unreddened F-type stars shows that HD 35929 is slightly redder for its spectral type (F2) than may be attributed to the interstellar extinction. On the other hand, the absence of the DIBs in its spectrum puts an upper level for the reddening at $A_{\mathrm{V}} \sim 0.1 \mathrm{mag}$.

Taking this slight reddening into account and using the relationships between the stellar temperature $\left(T_{\text {eff }}\right)$ and the Strömgren color index $b-y$ and between $T_{\text {eff }}$ and the Johnson color index $B-V$ from Napiwotzki et al. (1993), one can 
Table 2. Color indices of HD 35929 in the Johnson and Strömgren systems.

\begin{tabular}{ccccc}
\hline \hline Johnson & $U-B$ & $B-V$ & $V-R$ & $R-I$ \\
\hline & 0.14 & 0.42 & 0.37 & 0.27 \\
\hline Strömgren & $b-y$ & $\mathrm{~m}_{1}$ & $\mathrm{c}_{1}$ & $\beta$ \\
\hline & 0.266 & 0.168 & 0.767 & 2.693 \\
\hline
\end{tabular}

Listed are averaged data for the star's brightest state from papers cited above. The uncertainties are 0.01 mag for the Johnson data and 0.004 mag for the Strömgren data. The Walraven and Vilnius data are not presented, because each set of these data was published only in one paper.

calculate $T_{\text {eff }} \sim 6800 \mathrm{~K}$ for HD 35929. Furthermore, the Johnson $U-B$, the Strömgren $c_{1}$, and the Vilnius $U-P$ and $P-X$ color indices are sensitive to the luminosity and therefore the surface gravity. All these color indices consistently indicate that HD 35929 has a visual absolute magnitude $M_{\mathrm{V}} \sim 1 \mathrm{mag}$ and $\log g \sim 3.5$. We estimated these parameters comparing the color indices of HD 35929 from Table 2 (applying a reddening correction of $E(b-y)=0.04 \mathrm{mag}$, see below) with those of bright (not reddened) stars, whose fundamental parameters were derived spectroscopically by other authors (e.g., Erspamer \& North 2003; Gray et al. 2003). Since these color indices are known to contain some effects of metallicity and rotation (e.g., Gray \& Garrison 1989), we only used stars with projected rotational velocities $v \sin i \leq 100 \mathrm{~km} \mathrm{~s}^{-1}$ and metallicities $-0.3 \leq[\mathrm{Fe} / \mathrm{H}] \leq 0.1$. As we show below, the fundamental parameters of HD 35929 lie within these intervals. To illustrate our luminosity estimate, we plotted the Strömgren $b-y$ of the comparison stars versus $M_{\mathrm{V}}$ (Fig. 2).

With these estimates in hand, we compared the spectrum of HD 35929 with those of stars with similar $T_{\text {eff }}$ and $\log g$. The comparison spectra were taken from the ELODIE archive $^{2}$. The best match turned out to be $\beta$ Cas $\left(T_{\text {eff }}=6792 \mathrm{~K}, \log g=3.59\right.$, $v \sin i=74 \mathrm{~km} \mathrm{~s}^{-1},[\mathrm{Fe} / \mathrm{H}]=-0.15$, see Fig. 1). The spectral lines of $31 \mathrm{Cep}$, a cooler star with the same gravity $\left(T_{\mathrm{eff}}=\right.$ $\left.6637 \mathrm{~K}, \log g=3.59, v \sin i=81 \mathrm{~km} \mathrm{~s}^{-1},[\mathrm{Fe} / \mathrm{H}]=0.09\right)$, turned out to be stronger than those of HD 35929 which is partly an abundance effect. On the other hand, the spectra of HR $6987\left(T_{\text {eff }}=6745 \mathrm{~K}, \log g=4.13, v \sin i=73 \mathrm{~km} \mathrm{~s}^{-1}\right.$, $[\mathrm{Fe} / \mathrm{H}]=-0.12)$ and $\zeta \operatorname{Ser}\left(T_{\mathrm{eff}}=6698 \mathrm{~K}, \log g=4.08\right.$, $\left.v \sin i=71 \mathrm{~km} \mathrm{~s}^{-1},[\mathrm{Fe} / \mathrm{H}]=-0.10\right)$ with a higher gravity have lines consistently weaker than those of HD 35929. All the comparison star parameters are from Erspamer \& North (2003), whose data are consistent with those of other authors (e.g., Gray et al. 2003).

We retrieved UV spectra of HD 35929 and $\beta$ Cas from the IUE archive and compared continuum fluxes with each other and with Kurucz (1993) models. The results shown in Fig. 3 confirm that $T_{\text {eff }}$ of HD 35929 is close to $6800 \mathrm{~K}$. The spectral energy distribution (SED) of HD 35929 deviates from that of $\beta$ Cas longward of $\sim 0.8 \mu \mathrm{m}$ due to the IR excess, which is discussed in Sect. 4.

\footnotetext{
2 Available from http://atlas.obs-hp.fr/elodie/
}

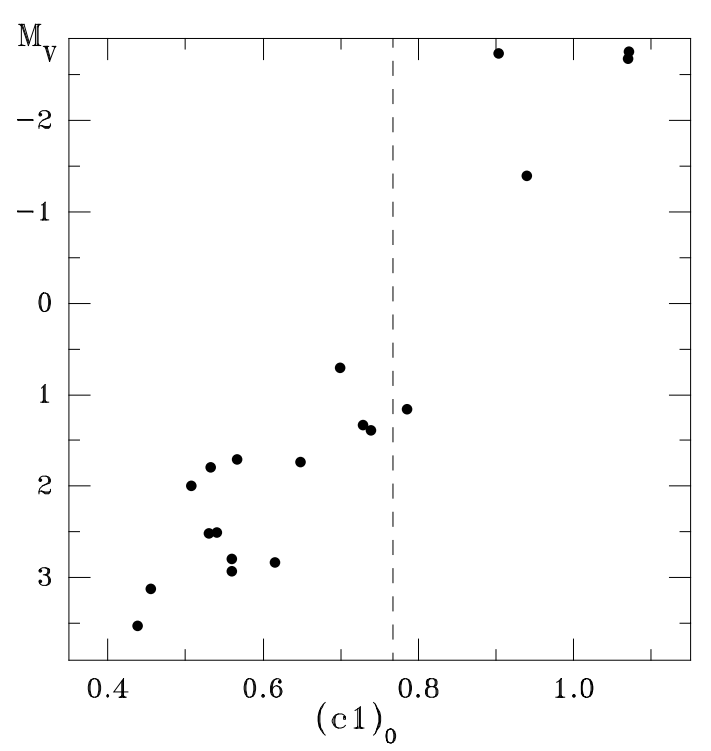

Fig. 2. The relationship of the reddening-corrected Strömgren color index $c_{1}$ and absolute visual magnitude $\mathrm{M}_{\mathrm{V}}$ for early F-type stars (F0-F4). Distances of all the comparison stars were taken from the HIPPARCOS catalog (ESA 1997). The dashed line indicates the color of HD 35929.

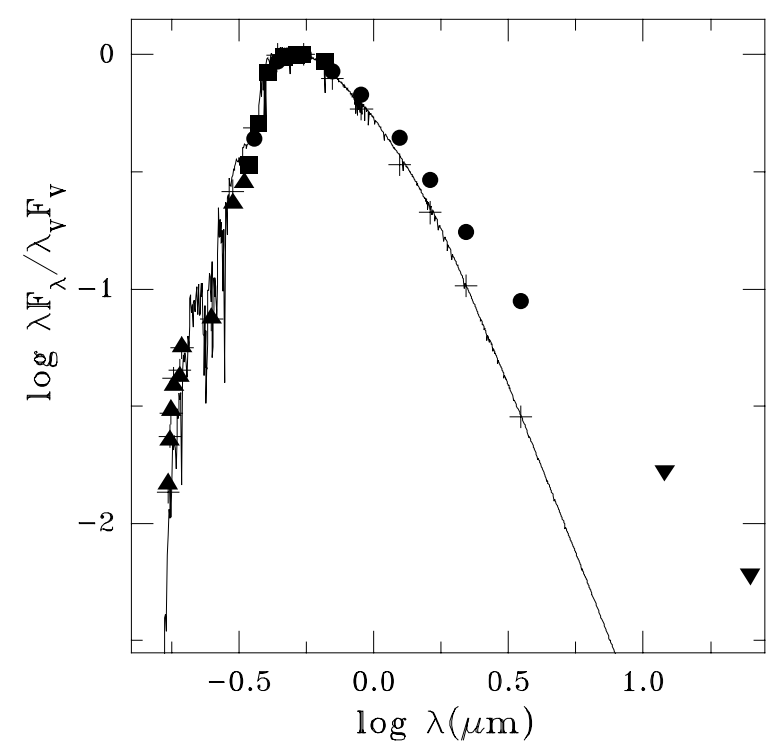

Fig. 3. The spectral energy distributions of HD 35929 and $\beta$ Cas. The IUE data for HD 35929 (continuum fluxes averaged within $100 \AA$ Antervals) are shown by upward triangles, the Vilnius system data by squares, the Johnson $U B V R I J H K L M$ data by circles, and the IRAS data by downward triangles. The data for $\beta$ Cas from the same sources and photometric systems are shown by crosses. The theoretical SED for $T_{\text {eff }}=6750 \mathrm{~K}, \log g=3.5$, and $60 \%$ of the solar metallicity from Kurucz (1993) is shown by the solid line. The fluxes are normalized to that in the Johnson $V$-band at $0.55 \mu \mathrm{m}$.

We used the SIMPLEX method (Gray et al. 2001) to fit the SED of HD 35929 in the range $0.2-0.5 \mu \mathrm{m}$ using the dereddened optical photometry and the IUE spectrum LWP27222LL as well as the optical spectrum of the star. The synthetic spectra were calculated using the program SPECTRUM (Gray \& Corbally 1994). The best fit shown in Fig. 4 was obtained 

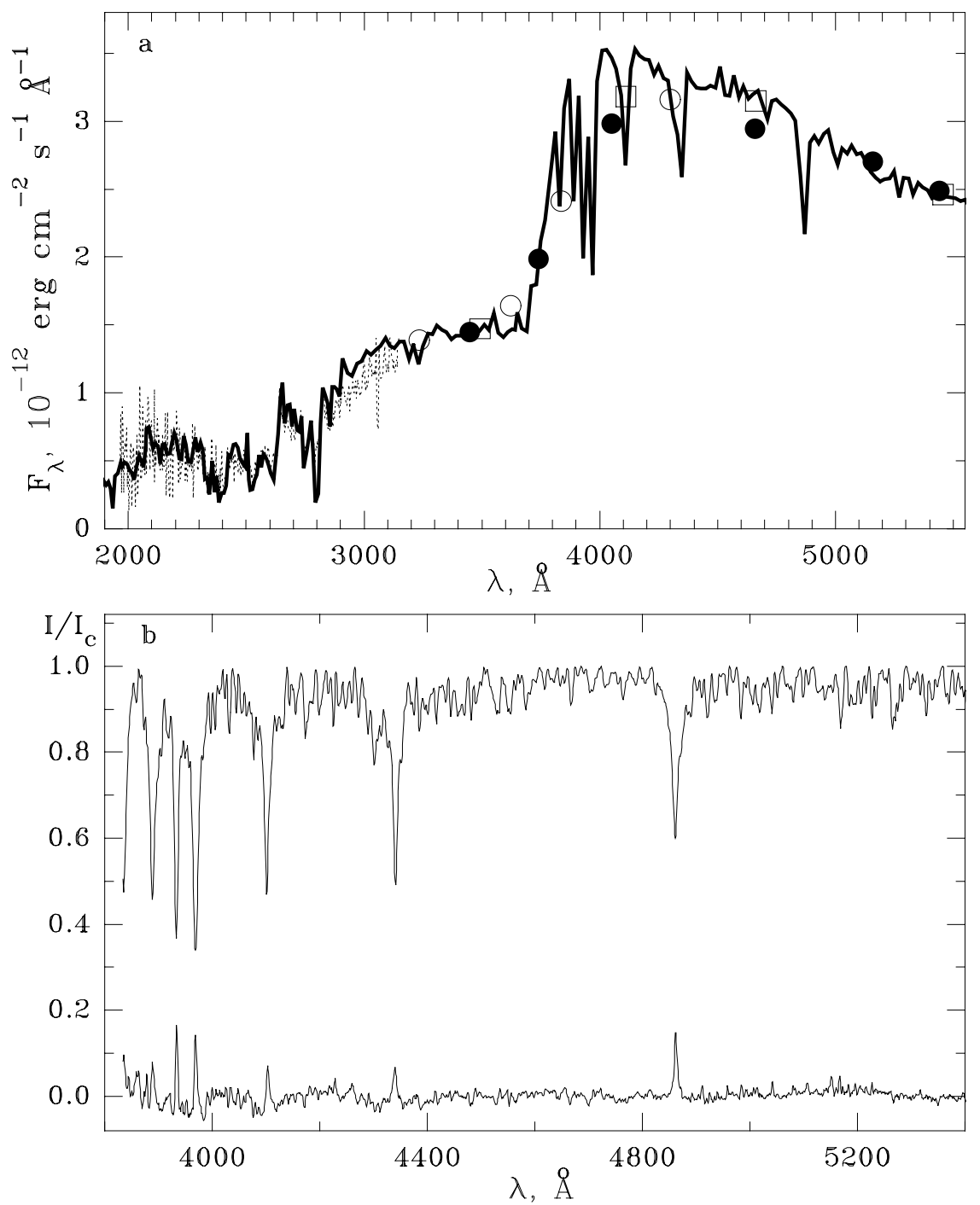

Fig. 4. The best fit for the spectral energy distributions and optical spectrum of HD 35929 derived using the SIMPLEX method. Panel a) represents the dereddened SED of HD 35929 composed from the IUE spectrum (thin dashed line), the Walraven photometry (open circles), the Vilnius photometry (filled circles), and the Strömgren photometry (open squares). The theoretical SED is shown by the thick solid line. The upper line in panel b) represents the low-resolution spectrum of HD 35929 obtained at the Dark Sky Observatory of Appalachian State University (Gray \& Corbally 1998). The lower line shows the difference between the observed spectrum and theoretical fit. The intensity is normalized to the continuum level.

for $T_{\text {eff }}=6880 \mathrm{~K}, \log g=3.3$, the microturbulent velocity $v_{\mathrm{t}}=3.5 \mathrm{~km} \mathrm{~s}^{-1}$, and $E(b-y)=0.04$ mag (which corresponds to $A_{\mathrm{V}}=0.17 \mathrm{mag}$ ) with uncertainties of $100 \mathrm{~K}, 0.1,0.2 \mathrm{~km} \mathrm{~s}^{-1}$, and $0.01 \mathrm{mag}$, respectively. The high-resolution optical spectrum is also fitted well with these parameters.

Combining the derived $T_{\text {eff }}$ and $\log g$ with the observed brightness and comparing them with the evolutionary tracks by Schaller et al. (1992), we found that they correspond to an initial mass $M / M_{\odot}=2.3 \pm 0.2$, a luminosity $\log L / L_{\odot}=1.7 \pm 0.2$, and a distance $D=345 \pm 60 \mathrm{pc}$. With this temperature and luminosity, HD 35929 is located beyond the main-sequence. The other results of the spectroscopic comparison were the measurements of $v \sin i=70 \pm 5 \mathrm{~km} \mathrm{~s}^{-1}$ and $[\mathrm{Fe} / \mathrm{H}]=-0.2 \pm 0.1$.

Investigation of the optical and near-IR (from the 2MASS catalog, Cutri et al. 2003) colors of stars close to HD 35929 on the sky (within $1^{\circ}$ ) shows that the interstellar extinction is smaller than $A_{\mathrm{V}} \sim 0.1 \mathrm{mag}$ at distances of at least $500 \mathrm{pc}$ from the Sun. This is consistent with our distance estimate from the star's fundamental parameters and the evolutionary tracks.

A similar comparison with the pre-main-sequence evolutionary tracks by Palla \& Stahler (1993) gives $M / M_{\odot}=3.6 \pm$ $0.2, \log L / L_{\odot}=2.1 \pm 0.2$, and $D=510 \pm 120 \mathrm{pc}$. With these parameters, HD 35929 is placed virtually on the birthline for intermediate-mass stars. In Sect. 4 we show that this is an unlikely situation.

\subsection{Circumstellar matter}

CS matter usually manifests itself by an emission-line spectrum and an IR excess. For HD35929 a few emission lines have been reported (e.g., $\mathrm{Mg}$ II, C IV, and $\mathrm{O}$ I in the UV and $\mathrm{H} \alpha$ ) in addition to a weak IR excess. The $\mathrm{Mg}$ II lines 


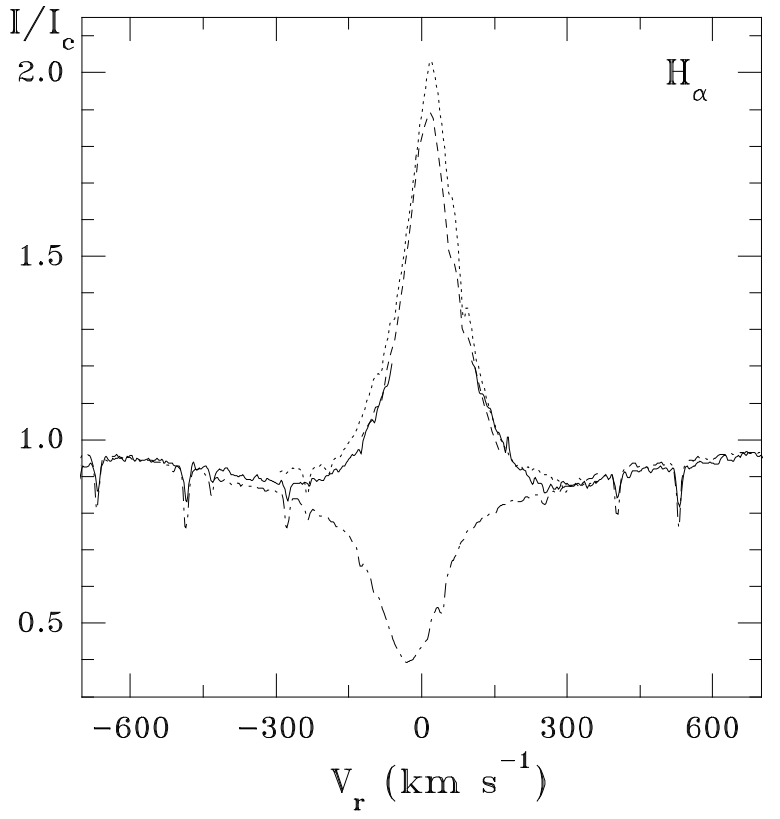

Fig. 5. The $\mathrm{H} \alpha$ line profile in the spectrum of HD 35929. The solid line shows the ELODIE spectrum in which the emission peak region is corrupted. The dotted line represents the $2001 \mathrm{McDonald}$ spectrum and the dashed line represents the $2002 \mathrm{McDonald}$ spectrum. The dashdotted line shows the spectrum of $\beta$ Cas. The heliocentric radial velocities are given in $\mathrm{km} \mathrm{s}^{-1}$, and intensities are normalized to the underlying continuum.

at 2796 and $2803 \AA$ have double-peaked profiles with peak separation of $\sim 150 \mathrm{~km} \mathrm{~s}^{-1}$ (shown in Grady et al. 1996). The lines of C IV at $1549 \AA$ and O I at $1302 \AA$ were detected only in the low-resolution mode, and their profiles were not resolved. The $\mathrm{H} \alpha$ line has a single-peaked profile (full width at halfmaximum $F W H M \sim 120 \mathrm{~km} \mathrm{~s}^{-1}$, full width at the photospheric level $F W Z I \sim 600 \mathrm{~km} \mathrm{~s}^{-1}$, peak intensity $I_{\max } / I_{\mathrm{c}}=1.9 \pm 0.1$, equivalent width $E W=3.2 \pm 0.1 \AA$ ) and show small variations in our spectra (Fig. 5).

The Mg II lines form mostly in the chromosphere and, as a matter of fact, have very similar appearance to those of normal F type stars (Böhm-Vitense et al. 2001). Thus, this feature is not descriptive of the type of CS matter distribution. The O I and Si IV emission lines in the UV region reported by Grady et al. (1996) may also have chromospheric origin. The $\mathrm{H} \alpha$ line profile suggests that the CS matter is distributed non-spherically near the star, the optical depth in the line of sight is small, and the plane of the preferential CS matter concentration is viewed close to pole-on (a spherical distribution would result in a $\mathrm{P}$ Cyg type $\mathrm{H} \alpha$ line profile, while an edge-on disk would produce a double-peaked profile; e.g., Meeus et al. 1998).

The IR excess of HD 35929 is one of the weakest among the Herbig Ae/Be star candidates. To illustrate this, we calculated the excess in the $K$-band $(2.2 \mu \mathrm{m})$ for all known late A-type - F-type pre-main-sequence star candidates with wellestablished fundamental parameters and reddening and plotted it against the mid-IR SED slope (Fig. 6). The $K$-band excess is derived as a difference between the dereddened observed flux and the photospheric flux (in magnitudes) using the intrinsic

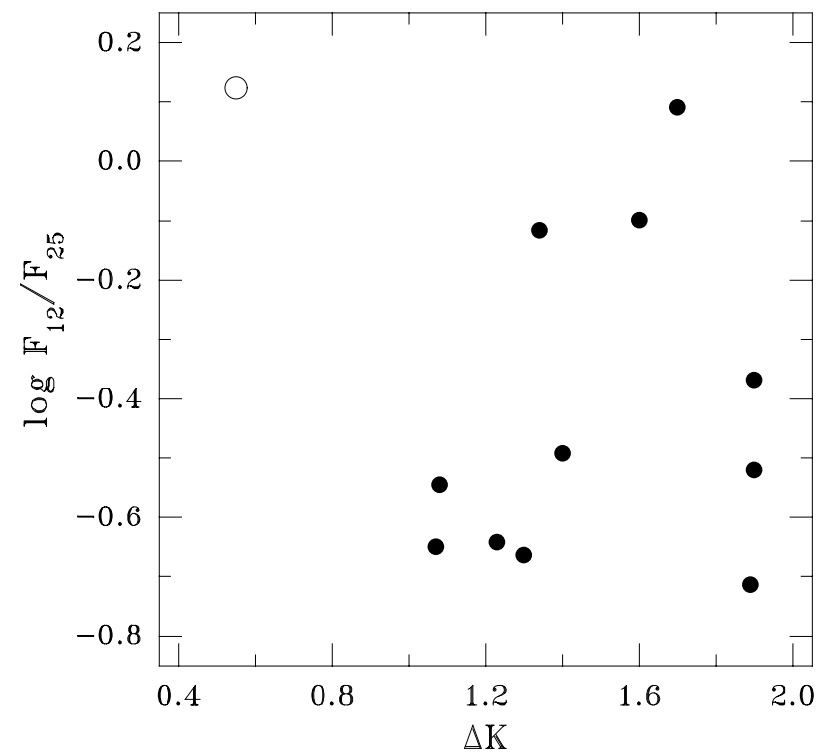

Fig. 6. The color-excess characteristics of late A-type - early F-type pre-main-sequence star candidates (from Thé et al. 1994). The data for HD 35929 are shown by an open circle, with filled circles for other stars. The $K$-band excess (see text) is given in magnitudes, and the IRAS flux ratio is dimensionless (the fluxes at 12 and $25 \mu \mathrm{m}$ are taken in Jy).

color indices by Bessell et al. (1998). It is seen that HD 35929 has the smallest $K$-band excess as well as the largest ratio of the 12 and $25 \mu \mathrm{m}$ fluxes $\left(F_{12} / F_{25}\right)$. The latter, in combination with the undetected $60 \mu \mathrm{m}$ flux, implies a lack of cold dust, in contrast to the SEDs of the other pre-main-sequence objects.

The small IR excess suggests that the CS dust is optically thin. To model it, we used the $1 \mathrm{D}$ radiation transfer code DUSTY (Ivezić et al. 1999). The stellar parameters derived above and optical properties of the mean interstellar dusty particles (Draine \& Lee 1984) were assumed. A very good fit was found for the following parameters: the dust optical depth in the $V$-band $\tau_{V}=0.25$, the dust sublimation temperature $T_{\text {sub }}=1500 \mathrm{~K}$, the radial density distribution $\rho_{d} \sim r^{-2}$, the interstellar extinction $A_{\mathrm{V}}=0.1 \mathrm{mag}$ (Fig. 7). The radial extent of the dusty envelope cannot be well constrained owing to the lack of far-IR data. However, the observed $F_{12} / F_{25}$ ratio is consistent with the absence of dust particles cooler than $\sim 100 \mathrm{~K}$. The strong silicate emission band was not detected by the IRAS satellite, as the star's flux at $12 \mu \mathrm{m}$ is well below the threshold of the IRAS Low-Resolution Spectrograph. However, it should be easily detectable by the Spitzer Space Observatory.

Since the dust is optically thin, its exact spatial distribution is irrelevant. Also, as we showed earlier (Miroshnichenko et al. 1997b), the CS reddening is very weak at small $\tau_{V}$ due to the scattering effect. Thus, our DUSTY modeling results do not contradict the $E(b-y)=0.04$ mag obtained from the SED and spectrum modeling with the SIMPLEX method. Furthermore, models with a density distribution slope of 1.5 (usually used for accretion disks of Herbig Ae/Be stars) produce too little near-IR emission even at the highest possible $T_{\text {sub }}=1500 \mathrm{~K}$ 


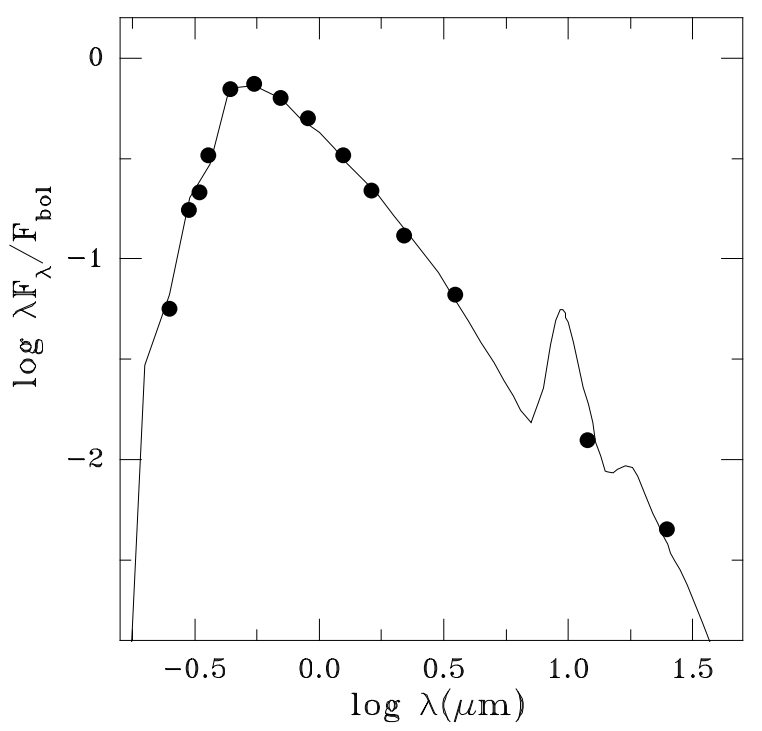

Fig. 7. The model fit to the spectral energy distribution of HD 35929. The data for HD 35929 are shown by filled circles. The theoretical SED with the parameters described in the text is shown by the solid line. The fluxes are normalized to the model bolometric flux.

for silicate particles. The slope of 2 found here is consistent with a steady-state stellar wind.

\section{Discussion}

The results presented here show that both the photometric data and the absorption-line spectrum of HD 35929 in the optical region are consistent with a classification as a normal F-type giant. The mild metal deficiency is not unusual among these objects (e.g., Erspamer \& North 2003). However, the $\mathrm{H} \alpha$ emission and IR excess make the star special. Its location in the direction of the Orion OB1 association (near the southern edge of this star formation region, in the Sword region defined by Blaauw 1964) raised a suspicion that it is a pre-main-sequence star (see Sect. 1). Its small proper motion (see the USNO-B1.0 catalog, Monet et al. 2003) does not contradict the object's membership in this aggregate, but our spectroscopic distance is significantly smaller than that derived for the Sword region from the HIPPARCOS data $(506 \pm 37 \mathrm{pc}$, de Zeeuw et al. 1999). Thus, HD 35929 turns out likely to be a foreground object for Ori OB1.

Furthermore, such a small IR excess and lack of cold dust are not common in Herbig Ae/Be stars. Studies of the IR excess evolution show that the near-IR excess usually disappears first as the star evolves toward the main sequence (e.g., Miroshnichenko et al. 1996; Malfait et al. 1998). The shape of the IR excess is more consistent with a steady-state mass loss than with accretion. Given the star's weak wind (from the weak $\mathrm{H} \alpha$ emission), it is hard to explain why the coldest part of the CS disk is lost. Also, the small IR excess contradicts the position of HD 35929 near the birthline for Herbig Ae/Be stars, where they are expected to be surrounded by a significant amount of CS matter.

On the other hand, it is not clear how the dust has formed near the star. The CS matter density is probably too small

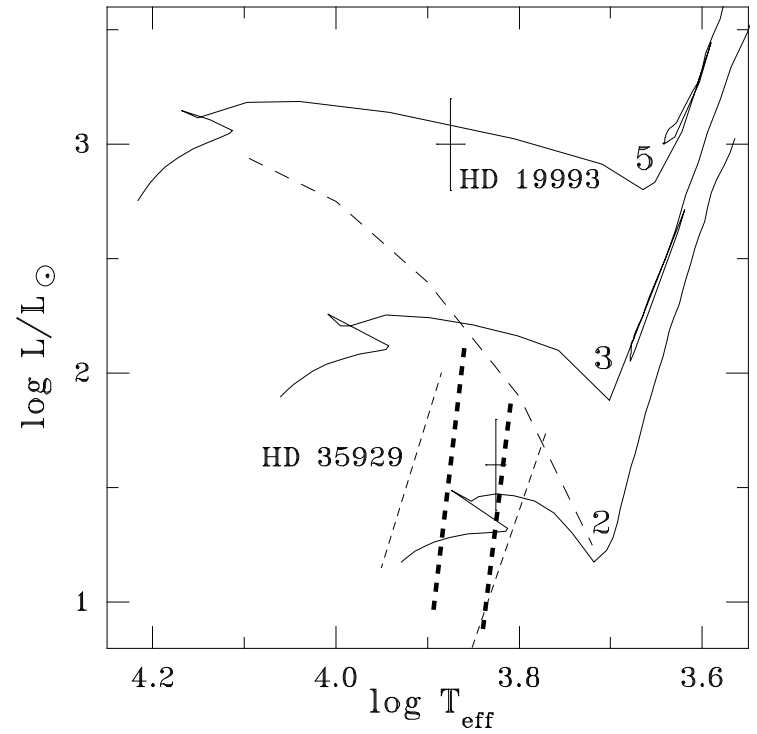

Fig. 8. Part of the Hertzsprung-Russell diagram with the positions of HD 19993 and HD 35929 indicated. The evolutionary tracks from Schaller et al. (1992) are shown by solid lines. Numbers near the tracks indicate the corresponding main-sequence masses in solar units. The birthline for pre-main-sequence intermediate-mass stars by Palla \& Stahler (1993) is shown by a long-dashed line. The theoretical boundaries of the instability strip for pre-main-sequence stars by Marconi \& Palla (1998) are shown by thick short-dashed lines, and those for evolved $\delta$ Sct stars from Breger \& Pamyatnykh (1998) by thin shortdashed lines.

at $\sim 70$ stellar radii, where $T_{\text {sub }}$ is reached. Another example of this situation is HD 19993 (A7/8 II) which exhibits weak double-peaked $\mathrm{H} \alpha$ emission and an IR excess, very similar to that of HD 35929, and has also passed the main-sequence stage (Miroshnichenko et al. 2003, see Fig. 8). Together they might represent a group of evolved dust-forming intermediate-mass stars. No other stars with such properties have been identified yet, probably because of the low sensitivity of the IRAS survey and a lack of high-resolution spectroscopic surveys, which are capable of detecting weak line emission. More such stars might be found by moderate or high-resolution spectroscopy $(R \geq 10000)$ of early-type stars with IR excesses (from analysis of such catalogs as 2MASS, IRAS, and MSX).

The dust formation mechanism in the intermediate-mass hot stars prior to the Asymptotic Giant Branch (AGB) evolutionary stage is not known yet. Some ideas have been suggested for massive stars with powerful winds (Wolf-Rayet stars, B[e] supergiants; see, e.g., Williams et al. 2001 and Kraus \& Lamers 2003). In principle, HD 35929 might have an undetected companion, responsible for the IR excess. However, there are no traces of such a companion either in the HIPPARCOS astrometric data (V.V. Makarov, priv. commun.) or in our spectroscopic data.

The derived fundamental parameters place HD 35929 within the instability strips for both evolved $\delta$ Sct and premain-sequence stars (Fig. 8). $\beta$ Cas, with an almost identical absorption-line spectrum, is also known to exhibit periodic brightness variations of the $\delta$ Sct type. Such variations, with an amplitude of $0.02 \mathrm{mag}$ and a period of $0.196 \pm 0.005$ day, were 
found in HD 35929 by Marconi et al. (2000). Using a periodluminosity-temperature relationship for $\delta$ Sct stars from López de Coca et al. (1990), one can derive a bolometric luminosity $\left(\log L / L_{\odot}\right)$ of $1.42,1.57$, and 1.72 for pulsation in the fundamental mode, first, and second overtone, respectively. Our spectroscopic data are consistent with these estimates.

Although some other pre-main-sequence candidates were found to exhibit $\delta$ Sct type variations (e.g., V351 Ori, HR 5999, HD 142666, see Catala 2003 and Marconi \& Palla 2003), this fact alone, or even in combination with the presence of an IR excess and an emission-line spectrum, cannot be used to determine the object's evolutionary state. Perhaps detection of evolutionary period changes, which were predicted to be 10-100 times faster for the pre-main-sequence $\delta$ Sct pulsators by Breger \& Pamyatnykh (1998), might help to resolve this issue.

The results of our spectroscopic study of HD 35929 can be summarized as follows:

1. The absorption-line spectrum suggests an MK type F2 III, with $\beta$ Cas being a very close match across the entire observed wavelength range. A weak interstellar reddening of $E(b-y)=0.04$ mag was found.

2. We derived the projected rotational velocity $v \sin i=70 \pm$ $5 \mathrm{~km} \mathrm{~s}^{-1}$ and measured the radial velocity $V_{\mathrm{r}}=20.7 \pm$ $1.6 \mathrm{~km} \mathrm{~s}^{-1}$, which turned out to be unchanging with time.

3. The emission component of the $\mathrm{H} \alpha$ line was almost constant in our 3 spectra obtained in 2001-2003.

4. The fundamental parameters, accurately derived for the first time, are consistent with the location of the star in the foreground of the Ori OB1 association, at a distance of $\sim 350 \mathrm{pc}$. The IR excess is too small and its shape is very different from that of pre-main-sequence stars. We suggest that the star has evolved beyond the main sequence; however, the nature of the IR excess remains puzzling.

Acknowledgements. Miroshnichenko and Bjorkman acknowledge support from NASA grant NAG5-8054. Karen Bjorkman is a Cottrell Scholar of the Research Corporation, and gratefully acknowledges their support. This publication has made use of the SIMBAD database operated at CDS, Strasbourg, France, and of data products from the Two Micron All Sky Survey, which is a joint project of the University of Massachusetts and the Infrared Processing and Analysis Center/California Institute of Technology, funded by the National Aeronautic and Space Administration and the National Science Foundation. It is also partly based on INES data from the IUE satellite.

\section{References}

van den Ancker, M. A., de Winter, D., \& Tjin A Dje, H. R. E. 1998, A\&A, 330, 145

Bessell, M. S., Castelli, F., \& Plez, B. 1998, A\&A, 333, 231

Blaauw, A. 1964, ARA\&A, 2, 213

Böhm-Vitense, E., Mena-Werth, J., Carpenter, K. G., \& Robinson, R. D. 2001, ApJ, 550, 457

Breger, M., \& Pamyatnykh, A. 1998, A\&A, 332, 958

Catala, C. 2003, Ap\&SS, 284, 53

Corporon, P., \& Lagrange, A.-M. 1999, A\&AS, 136, 429
Cutri, R. M., Skrutskie, M. F., Van Dyk, S., et al. 2003, 2MASS AllSky Catalog of Point Sources, University of Massachusetts and Infrared Processing and Analysis Center/California Institute of Technology

Draine, B. T., \& Lee, H. M. 1984, ApJ, 285, 89

Eimontas, A., \& Sudzius, J. 1998, Balt. Astr., 7, 407

Erspamer, D., \& North, P. 2003, A\&A, 398, 1121

ESA 1997, The Hipparcos and Tycho Catalogues ESA SP-1200

de Geus, E. J., Lub, J., \& van de Grift, E. 1990, A\&AS, 85, 915

Grady, C. A., Pérez, M. R., Talavera, A., et al. 1996, A\&AS, 120, 157

Gray, R. O., \& Garrison, R. F. 1989, ApJS, 69, 301

Gray, R. O., \& Corbally, C. J. 1994, AJ, 107, 742

Gray, R. O., \& Corbally, C. J. 1998, AJ, 116, 2530

Gray, R. O., Graham, P. W., \& Hoyt, S. R. 2001, AJ, 121, 2159

Gray, R. O., Corbally, C. J., Garrison, R. F., McFadden, M. T., \& Robinson, P. E. 2003, AJ, 126, 2048

Ivezić, Ž., Nenkova, M., \& Elitzur, M. 1999, User Manual for DUSTY, Univ. of Kentucky Internal Report, accessible at http://www. pa.uky.edu/ moshe/dusty

Kraus, M., \& Lamers, H. J. G. L. M. 2003, A\&A, 405, 165

Kurucz, R. L. 1993, CD-ROM No. 13, ATLAS9 Stellar Atmosphere Programs and $2 \mathrm{~km} \mathrm{~s}^{-1}$ Grid, Cambridge, Smithsonian Astrophysical Obs.

López de Coca, P., Rolland, A., Rodríguez, E., \& Garrido, R. 1990, A\&AS, 83, 51

Malfait, K., Bogaert, E., \& Waelkens, C. 1998, A\&A, 331, 211

Marconi, M., \& Palla, F. 1998, ApJ, 507, L141

Marconi, M., \& Palla, F. 2003, Ap\&SS, 284, 245

Marconi, M., Ripepi, V., Alcalá, J. M., et al. 2000, A\&A, 355, L35

McCarthy, J. K., Sandiford, B. A., Boyd, D., \& Booth, J. 1993, PASP, 105,881

Meeus, G., Waelkens, C., \& Malfait, K. 1998, A\&A, 329, 131

Miroshnichenko, A. S., Bergner, Yu. K., Kuratov, K. S., Mukanov, D. B., \& Sheikina, T. A. 1996, Astron. Rep., 40, 509

Miroshnichenko, A. S., Bergner, Yu. K., \& Kuratov, K. S. 1997a, Astron. Lett., 23, 97

Miroshnichenko, A. S., Ivezić, Ž., \& Elitzur, M. 1997b, ApJ, 475, L41

Miroshnichenko, A. S., Bjorkman, K. S., Chentsov, E. L., et al. 2001, A\&A, 377, 854

Miroshnichenko, A. S., Kusakin, A. V., Bjorkman, K. S., et al. 2003, A\&A, 412, 219

Monet, D. G., Levine, S. E., Casian, B., et al. 2003, AJ, 125, 984

Napiwotzki, R., Schönberner, D., \& Wenske, V. 1993, A\&A, 268, 653

Olsen, E. H. 1983, A\&AS, 54, 55

Olsen, E. H. 1994, A\&AS, 106, 257

Oudmaijer, R. D., van der Ween, W. E. C. J., Waters, L. B. F. M., et al. 1992, A\&AS, 96, 625

Palla, F., \& Stahler, S. 1993, ApJ, 418, 414

Panchuk, V. E., Piskunov, N. E., Klochkova, V. G., Yushkin, M. V., \& Ermakov, S. V. 2002, Preprint Special Astrophys. Observ., No. 170

Perry, C. L. 1991, PASP, 103, 494

Schaller, G., Schaerer, D., Meynet, G., \& Maeder, A. 1992, A\&AS, 96, 269

Straizys, V. 1963, Vilnius Obs. Bull., 7, 1

Thé, P. S., de Winter, D., \& Pérez, M. R. 1994, A\&AS, 104, 315

Williams, P. M., Kidger, M. R., van der Hucht, K. A., et al. 2001, MNRAS, 324, 156

de Winter, D., van den Ancker, M. E., Maira, A., et al. 2001, A\&A, 380, 609

de Zeeuw, P. T., Hoogerwerf, R., de Bruijne, J. H. J., Brown, A. G. A., \& Blaauw, A. 1999, AJ, 117, 354 